\title{
A Motion Analysis in the Volleyball Spike - Part 1: Three-dimensional Kinematics and Performance
}

\author{
Ben Serrien $^{1, *}$, Jeroen Ooijen ${ }^{1}$, Maggy Goossens ${ }^{2,3}$, Jean-Pierre Baeyens ${ }^{1,2,3}$ \\ ${ }^{1}$ Department of Biomechanics, Free University of Brussels, Belgium \\ ${ }^{2}$ Department of ICT and Electronics, University of Antwerp, Belgium \\ ${ }^{3}$ Thim Van Der Laan University College, Switzerland
}

Copyright $\mathrm{C} 2016$ by authors, all rights reserved. Authors agree that this article remains permanently open access under the terms of the Creative Commons Attribution License 4.0 International License

\begin{abstract}
In this study, three-dimensional kinematics and performance were analyzed for male and female top level and junior elite volleyball players in a spike, which is an essential offensive volleyball action. Top level players had higher impact speeds and jumping height than junior elite players for both genders and the male players had higher impact speeds and jumping height than the female players for both levels of expertise. For the analysis of the three-dimensional kinematics of the pelvis, trunk, shoulder and elbow angles and angular velocities, Statistical Parametric Mapping was used. No differences were observed for the orientation angles of pelvis and trunk, indicating a set of invariant kinematics. The pelvis and trunk angular velocities on the other hand did show many interesting differences throughout the approach, cocking and acceleration phase of the spike. The shoulder and elbow angles and angular velocities also illustrated the different strategies used for generating high impact speeds. An important conclusion was that interaction effects between gender and level of expertise were mostly observed in variables from the shoulder and elbow, whereas pelvis and trunk velocities only presented significant main effects. These differences suggest how the motion pattern of the young elite athletes will change over the following years when they gradually make the change towards the highest competitive levels.
\end{abstract}

Keywords Volleyball, Spike, Statistical Parametric Mapping, 3D kinematics, Biomechanics

\section{Introduction}

This paper presents the first outcomes of a longitudinal follow-up of young elite volleyball players. These players are enrolled in the 'Topsportschool Volleyball', cooperation between the Flemish government and the Flemish Volleyball Federation to support elite youth volleyball students of 14-18 years. The students follow twenty hours of regular school classes supplemented with twenty hours of volleyball training. It is our goal to follow a cohort for four years during their time at the school, representing an important stage of physical and motor development, in order to observe the changes in motion patterning. This paper presents the outcome of the first measurements where the students were compared to top level players from the highest level in the Belgian competition. Part 1 presents the analysis of the three-dimensional kinematics and performance on a group level. Part 2 of this study uses a single-subject approach to data analysis and concerns coordination- and performance variability.

The volleyball spike is an essential skill which an average top level volleyball player performs about 40.000 times per year [1]. Consequently, insight in the mechanics of the spike motion is an important issue in developing training programs. Contrasting groups at different skill levels can be helpful to generate hypotheses about the evolution of young elite athletes. Understanding the change in kinematics of the spike can be helpful in developing age-appropriate training programs. The volleyball spike is a complex motion that can be characterized in 4 phases [2]: approach phase, arm cocking phase, acceleration phase and follow through phase. From the moment of take-off until striking the ball, energy and (angular) momentum of the total body are conserved physical quantities. This conservation constrains the motion, but still an immense number of possible motion patterns are available to the motor system. Observing kinematic differences between groups that are known to generate different ball speeds, can tell us important mechanical aspects of the motion. A group analysis has a downside in masking important information during the averaging process. No individual players' motion pattern is accurately represented by the mean, but it can still be useful for generating hypotheses and is easier to communicate to coaches. Previous work in volleyball biomechanics has analyzed correlations between angular kinematics and ball speed [3], [4], correlations between strength performance, body mass index and ball speed [5], the patterns and relative intensities of shoulder muscle activity during the spike [6], 
differences in 3D kinematics between players with and without shoulder injuries [7], spike differences between volleyball and beach volleyball [8], differences between several spike techniques [2], [9] and differences between a volleyball spike, handball throwing and tennis serves [10]. To our knowledge, no study has compared players of different skill level. Furthermore male and female players have not yet been compared. Both skill level and gender are important factors that need to be taken into account when designing training programs for coordination and strength. The paper from Wagner et al. [11] described the kinematics of the spike jump. One of the more important conclusions from Wagner et al. is that not only the take-off and subsequent phases, but also an optimal approach technique is necessary to reach a maximal jumping height (and thus more time for the execution of the spike). This is similar to chaotic systems where the final solution is highly dependent on initial conditions [12]. The fact that the entire motion is important and not only is some discrete parts mostly neglected in classical statistical analyses of biomechanical data. Recent developments in Statistical Parametric Mapping for one-dimensional (biomechanical) time series [13] are able to allow researchers to use the entire time series in the analysis (and not only data at selected discrete time points). This can yield a much broader understanding of the mechanics at play. The aim of this paper is to use Statistical Parametric Mapping to analyze differences in the three-dimensional spike kinematics of the pelvis, trunk and spike arm shoulder and elbow between male and female top level and junior elite volleyball players.

\section{Materials and Methods}

\subsection{Subjects}

Eight male professional volleyball players and ten female professional volleyball players were recruited from two top-level Belgian volleyball teams (Liga A) alongside eight male and eleven female elite youth volleyball players from the Topsportschool (TSS). The main anthropometric characteristics of the subjects are displayed in Table 1. All players were injury-free at the time of the measurements and had no injuries limiting them to perform training and competitions during the previous six months. This research was approved by the ethical commission of the Brussels University Hospital and all participants and their parents have read and signed the informed consent.

Table 1. Anthropometric and volleyball characteristics (means \pm SD)

\begin{tabular}{ccccc}
\hline & $\begin{array}{c}\text { Age } \\
\text { (years) }\end{array}$ & $\begin{array}{c}\text { Volleyball } \\
\text { experience (years) }\end{array}$ & Height $(\mathrm{cm})$ & $\begin{array}{c}\text { Weight } \\
(\mathrm{kg})\end{array}$ \\
\hline Elite & 23.33 & 14.67 & 197.67 & 92.22 \\
male & \pm 2.78 & \pm 2.40 & \pm 7.05 & \pm 4.47 \\
Elite & 24.23 & 13.89 & 182.87 & 70.87 \\
female & \pm 2.01 & \pm 1.98 & \pm 12.10 & \pm 8.65 \\
TSS & 15.05 & 6.33 & 178.65 & 71.02 \\
boys & \pm 0.76 & \pm 1.98 & \pm 4.05 & \pm 6.63 \\
TSS girls & 15.19 & 5.67 & 169.78 & 62.03 \\
& \pm 0.54 & \pm 1.67 & \pm 10.67 & \pm 4.77 \\
\hline
\end{tabular}

\subsection{Procedures}

The volleyball players were instructed to execute an individual 15 minute warm-up consisting of cardiovascular and volleyball specific exercises to prevent any injuries when performing the spike motions. Afterwards, 34 retro reflective markers $(14 \mathrm{~mm})$ were attached to the skin with double-sided tape (sacrum, T10, T7, C7, 2 markers on the sternum, bilateral: spina iliaca anterior superior, trochanter major, femoral epicondylus lateralis, malleolus lateralis, acromion, humeral epicondylus lateralis and medialis, olecranon, radial and ulnar processus styloideus, dorsal head of metacarpal 2 and 5). On the ball we placed 6 retro reflective tapes to observe its trajectory and to verify ball impact. The subjects performed the volleyball spike motions with an individually chosen run-up length towards a tossed-up ball by a setter. The setter was an ex-professional volleyball player in the Belgian highest competition and could deliver the ball very accurately to a certain point above the net. Other researchers have used a rope to hang up the ball [8], [10]. We chose not to use this approach to increase the ecological validity of the analysis since players are not used to perform the spike to a fixed ball hanging in space but to a moving ball. The players were instructed to perform maximal velocity spikes towards the central backside of the field (a $3 \mathrm{~m}$ by $3 \mathrm{~m}$ target around position 6). Players had to perform ten successful spikes (the spike was successful if the ball landed in the target zone and if player and setter agreed that it was a good set-up). The maximal number of trials necessary to obtain ten successful ones was thirteen and the mode was twelve.

\subsection{Data Collection and Data Processing}

Three-dimensional kinematic data were captured with a 6-camera VICON MX F20 system at $250 \mathrm{~Hz}$ (VICON Peak, Oxford UK). Three-dimensional marker trajectories were reconstructed and gaps were filled in the VICON Nexus 1.8.2 software and smoothed with a fourth order Butterworth filter (zero lag) at a cut-off frequency of $13 \mathrm{~Hz}$. Marker coordinates were exported to a .csv file and imported into a custom-made algorithm in Mathcad 14.0 (Parametric Technology Corporation, MA, USA). The origin of the global reference frame was set at the line under the net with the positive $\mathrm{Y}$-axis in the direction of the spike, the positive $\mathrm{X}$-axis to the right and an upward positive $\mathrm{Z}$-axis. The moment of ball impact was defined according to the definition given in [10] as the moment where the hand's horizontal acceleration decreased abruptly. At this frame, hand impact speed (midpoint of the metacarpal markers) was calculated and extracted for statistical analysis. Jumping height was defined as the maximal vertical position of the pelvis center-of-mass minus the standing vertical position obtained from a static calibration trial [14]. Local reference frames were defined for the upper-arm, lower-arm, the trunk and the pelvis. Shoulder joint angles were defined as the Euler angles between the upper arm and trunk reference 
frames in an order of horizontal ab/adduction, ab/adduction and endo/exorotation. Trunk and pelvis attitude was defined as the Cardan angles between the trunk frame and the global reference frame in an order of forward/backward tilt, lateral tilt and rotation. The elbow angle was calculated as the relative orientation of the upper-arm and the lower-arm longitudinal vectors. All angle-time series were differentiated with respect to time (central difference method) after the necessary transformations for the Euler/Cardan angles [15]. All variables $(n=20)$ were calculated within a time-span of $400 \mathrm{~ms}$ (100 data frames) before ball release and $80 \mathrm{~ms}$ (20 data frames) after ball release; this corresponds approximately to the lowest point of the center-of-mass before the take-off until the end of the follow-through phase. The Euler and Cardan rotation sequences yielded no gimbal locks during this time-span. All kinematic variables for the left-handed players were transformed so the kinematic time series showed the same anatomical interpretation as for all right-handed players. The time series from all ten trials were averaged per player to be used as input for the statistical analyses.

\subsection{Statistical Procedures}

The three-dimensional kinematics and performance parameters (hand impact speed and jumping height) were analyzed in a 2x2-ANOVA with level of expertise and gender as between subjects' factors. The performance variables ANOVAs were performed in the usual way for scalar (0D) data. Homogeneity of variance was tested with the Levene's test and normality of the data was assessed with a QQ-plot and Shapiro-Wilk test. The ANOVAs for the kinematic time series were executed with the open-source software package SPM-1D (1-dimensional statistical parametric mapping, www.spm1d.org; (C) T.C. Pataky). Statistical Parametric Mapping was originally developed in the field of neuro-imaging [16] and uses Random Field Theory (RFT) [17] to make inferential statistics. A test-statistic $\left(\mathrm{t}, \mathrm{F}, \mathrm{chi}^{2}, \ldots\right)$ is computed for every time point of the dataset, but instead of calculating a p-value for every time point, RFT is used to compute a threshold test-value (based on the alpha-level and smoothness of the data) and an overall p-value for supra-threshold clusters. It is important to note that SPM does not perform an inferential test at every time point of the dataset and thus avoids a large multiple testing problem. Instead, only an inference ( $p$-value calculation) is drawn about supra-threshold clusters. The correct interpretation of this p-value is that it is the probability that a random $1 \mathrm{D}$ Gaussian (or $\mathrm{t}, \mathrm{F}, \mathrm{chi}^{2}, \ldots$ ) time series with the same smoothness as the observed data would produce a supra-threshold cluster with an extent as large as the observed cluster [13]. SPM is a relatively new statistical technique in biomechanics that can be used to perform any kind of test from the general linear model (i.e. t-test, all kind of ANOVAs, regression, multivariate tests,) on time series data. In contrast to classical statistical testing, where extrema from a time series were extracted, this procedure does not introduce a bias by neglecting the largest part of the data and allows to examine non-directed research questions about any kind of 1D time series [13] without introducing a multiple testing problem. Additionally, it allows presenting the time series and statistics graphically and leads to a better interpretation. Due to the high number of variables (20), we divided the variables in 3 sets and performed a 1-way MANOVA on each set (2-way MANOVA procedures are presently not available). The first set of variables are the orientation angles of pelvis and trunk in the global reference frames $(n=6)$, the second set are the angular velocities of pelvis and trunk $(\mathrm{n}=6)$ and the third set are the angles and angular velocities of shoulder and elbow $(n=8)$. Post-hoc analyses on the variables from significant MANOVAs were executed in 2-way ANOVAs to see in which variables, the effects were located. A design-matrix for the unbalanced design was designed ad hoc. The two main effects and interaction effect were estimated by comparing the full and reduced design matrices. If the interaction effect was significant, the main effects could not be interpreted and post-hoc two-sample t-tests were performed to compare top level players with junior elite players for both genders separately. We chose to split the analysis on gender, because the primary aim was on skill level to generate hypotheses on how the junior elite players will evolve. Exact probability-values for the ANOVAs of unbalanced design are not available yet, but hypothesis testing $(p<\alpha)$ can be performed (probability-values for the t-tests and MANOVA are available). All statistical analyses were performed in Matlab R2015a and the level of significance was set at $\alpha=$ 0.05 . To control the multiple testing problem of the large set of dependent variables, Bonferroni corrections were made on this alpha level for all subsequent post-hoc tests. For univariate ANOVAs following a MANOVA with six and eight variables, this meant $\alpha=0.00833$ and $\alpha=0.00625$ respectively. For post-hoc t-tests following an ANOVA with a significant interaction effect, these alpha levels were further decreased to 0.004167 and 0.00313 respectively.

\section{Results}

A first important remark is the variability in the toss-up, because this could potentially influence the data. The variability in toss-up was quantified using the range on the location of ball impact and was calculated for each participant individually. The largest observed ranges over all participants were $15 \mathrm{~cm}, 19 \mathrm{~cm}$ and $18 \mathrm{~cm}$ in the anterior-posterior, left-right and superior-inferior direction respectively. These small variations in ball contact location had probably only little systematic influence on the movement execution and were necessary to reflect a realistic volleyball spike. Table 2 presents the performance analysis. As expected, top level players had a higher impact speed than junior elite players for both genders and male players had a higher impact speed than female players. The same observation can be made for the analysis of jumping height. 
The MANOVA on the orientation angles of the pelvis and trunk did not reach any significant level. For the pelvis and trunk angular velocities, three significant supra-threshold clusters are seen in the MANOVA (Figure 1, left): from the beginning of the data registration until $352 \mathrm{~ms}$ before ball impact $(\mathrm{p}=0.0011)$, from $220 \mathrm{~ms}$ until $164 \mathrm{~ms}$ before ball impact $\left(\mathrm{p} \sim 10^{-5}\right)$ and a larger one from $112 \mathrm{~ms}$ before until
$16 \mathrm{~ms}$ after ball impact $\left(\mathrm{p} \sim 10^{-13}\right)$. During these time frames, there is a significant difference in the velocity of pelvis and trunk between the four groups. The MANOVA on spike arm orientation and velocity variables yielded one large supra-threshold cluster from $48 \mathrm{~ms}$ before ball impact until the end of the registered data $\left(\mathrm{p} \sim 10^{-8}\right)$ (Figure 1, right).

Table 2. Means \pm SD of hand impact speed and jumping height and outcomes of the two-way ANOVAs

\begin{tabular}{|c|c|c|}
\hline \multicolumn{3}{|l|}{ Means \pm SD: } \\
\hline & Impact speed (m/s) & Jumping height $(\mathrm{cm})$ \\
\hline Top level, male & $16,272 \pm 1,234$ & $62,013 \pm 3,358$ \\
\hline Top level, female & $12,404 \pm 1,832$ & $48,510 \pm 4,545$ \\
\hline TSS, boys & $13,987 \pm 0,572$ & $53,763 \pm 6,324$ \\
\hline TSS, girls & $11,157 \pm 1,012$ & $47,521 \pm 7,335$ \\
\hline \multicolumn{3}{|c|}{ ANOVA Impact speed: } \\
\hline \multicolumn{3}{|c|}{ Levene's test: $p=0,189$, Shapiro-Wilk: $p=0,980$} \\
\hline \multicolumn{3}{|c|}{$\begin{array}{c}\text { Expertise }\left(\mathrm{F}=17,489, \mathrm{p}<0,001, \eta_{\text {partial }^{2}}=0,346, \text { power }=0,982\right), \text { Gender }\left(\mathrm{F}=62,905, \mathrm{p}<0,001, \eta_{\text {partial }^{2}}\right. \\
=0,656, \text { power }=0,999), \text { Interaction }\left(\mathrm{F}=1,514, \mathrm{p}=0,227, \eta_{\text {partial }}{ }^{2}=0,044, \text { power }=0,223\right)\end{array}$} \\
\hline \multicolumn{3}{|c|}{ ANOVA jumping height: } \\
\hline \multicolumn{3}{|c|}{ Levene's test: $p=0,376$, Shapiro-Wilk: $p=0,145$} \\
\hline \multicolumn{3}{|c|}{$\begin{array}{c}\text { Expertise }\left(\mathrm{F}=20,445, \mathrm{p}<0,001, \eta_{\text {partial }}{ }^{2}=0,383, \text { power }=0,992\right), \text { Gender }\left(\mathrm{F}=63,507, \mathrm{p}<0,001, \eta_{\text {partial }^{2}}\right. \\
\quad=0,658 \text {, power }=0,999) \text {, Interaction }\left(\mathrm{F}=0,798, \mathrm{p}=0,378, \eta_{\text {partial }}{ }^{2}=0,024, \text { power }=0,140\right)\end{array}$} \\
\hline
\end{tabular}
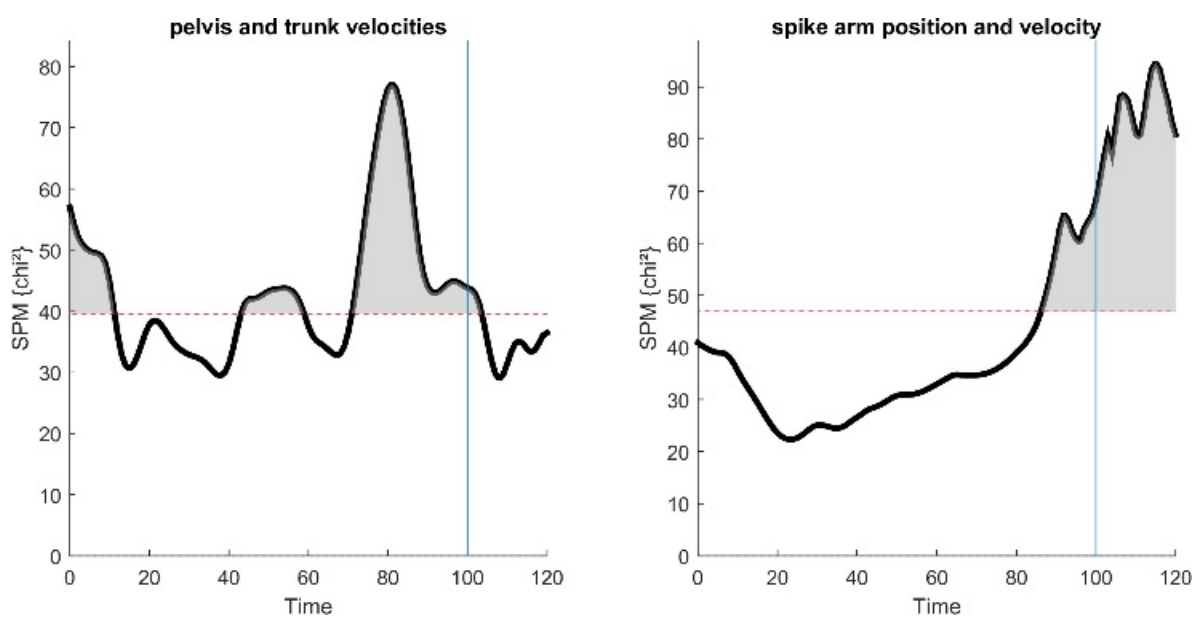

Figure 1. Results from the MANOVAs on pelvis and trunk velocities (left) and spike arm position and velocity (right). 
The following figures provide the time series (mean \pm SD-cloud) and the SPM two-way ANOVAs or post-hoc SPM t-tests on the kinematic variables from the two sets that yielded a significant MANOVA. The time span during which significant interaction effects occurred, are reported but the ANOVA figures are not shown to reduce the number of figures. The vertical lines at $t=100$ indicate the moment of ball impact. The horizontal lines indicate the threshold
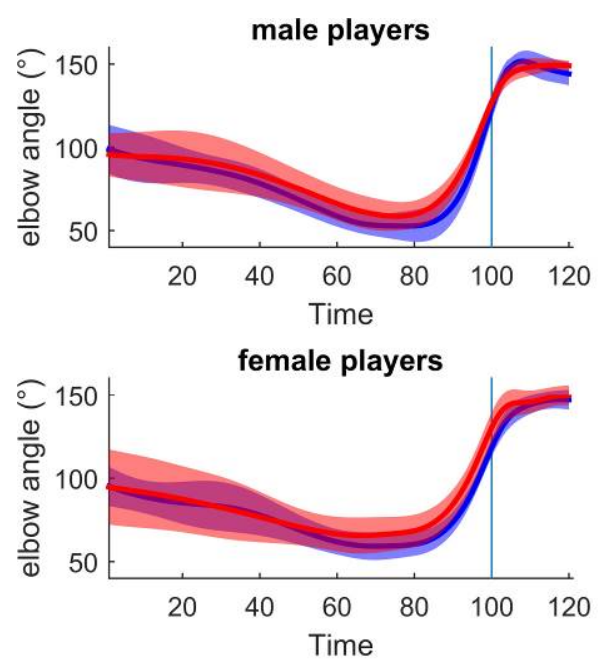

test-values ( $\mathrm{t} *$ or $\mathrm{F}^{*}$ values).

Figure 2 shows the results from the SPM 2-way ANOVA on the elbow angle time series. No significant interaction-effect or main effect of gender was observed. The time series showed a significant main effect of expertise on elbow angle from $36 \mathrm{~ms}$ until $4 \mathrm{~ms}$ before ball impact ( $\mathrm{p}<$ 0.00625 ). During this time span, the top level players had their elbow angle more extended.
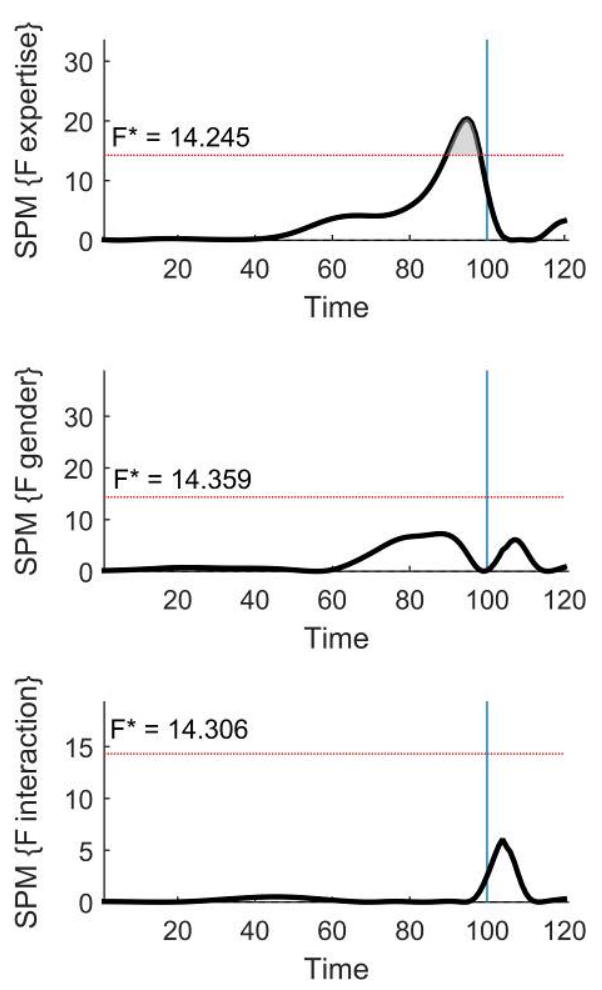

Figure 2. SPM two-way ANOVA on the elbow angle time series (red = top level players; blue $=$ junior elite players). $180^{\circ}$ represents full elbow extension.
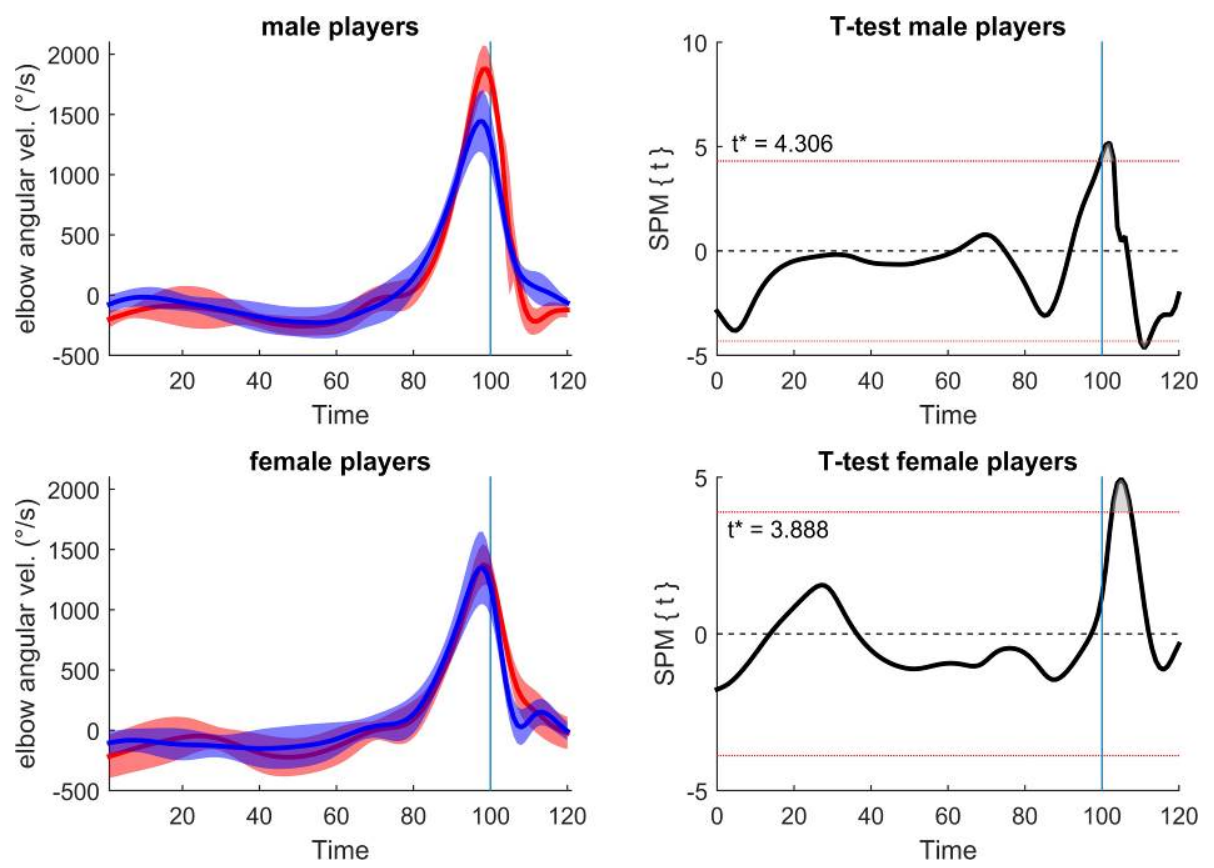

Figure 3. Post hoc T-tests on the elbow angular velocity time series (red $=$ top level players; blue $=$ junior elite players). Positive values are extension velocity and negative values are flexion velocity. 
Figure 3 presents the post-hoc SPM t-tests for the elbow angular velocity. An interaction effect in the SPM 2-way ANOVA was observed from $40 \mathrm{~ms}$ until $44 \mathrm{~ms}$ after ball impact $(p<0.00625)$. For the male players, the top level players had a significant higher elbow extension velocity from ball impact until $16 \mathrm{~ms}$ later $(\mathrm{p}=0.001)$ and $\mathrm{a}$ significant higher elbow flexion velocity from $40 \mathrm{~ms}$ until 52 $\mathrm{ms}$ after ball impact $(\mathrm{p}=0.00102)$. In the female players, the top level players showed a higher elbow extension velocity from $24 \mathrm{~ms}$ until $32 \mathrm{~ms}$ after ball impact $(\mathrm{p}=0.00234)$.

Figure 4 shows the post-hoc SPM t-tests on the shoulder rotation angles. Two interaction effects occurred for this variable: a first between $172 \mathrm{~ms}$ and $76 \mathrm{~ms}$ before ball impact $(p<0.00625)$ and a second between $12 \mathrm{~ms}$ after impact until the end of the registered data $(p<0.00625)$. The post-hoc tests revealed that there was no significant difference between the female players of different expertise. The male
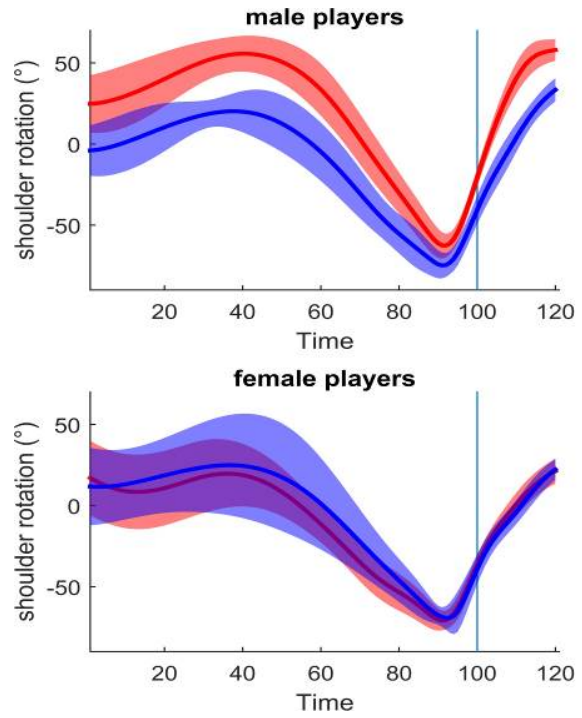

Figure 4. Post hoc $\mathrm{T}$-tests on the shoulder rotation angle time series ( $\mathrm{red}=$ top level players; blue $=$ junior elite players). Positive values are endorotation and negative values are exorotation.
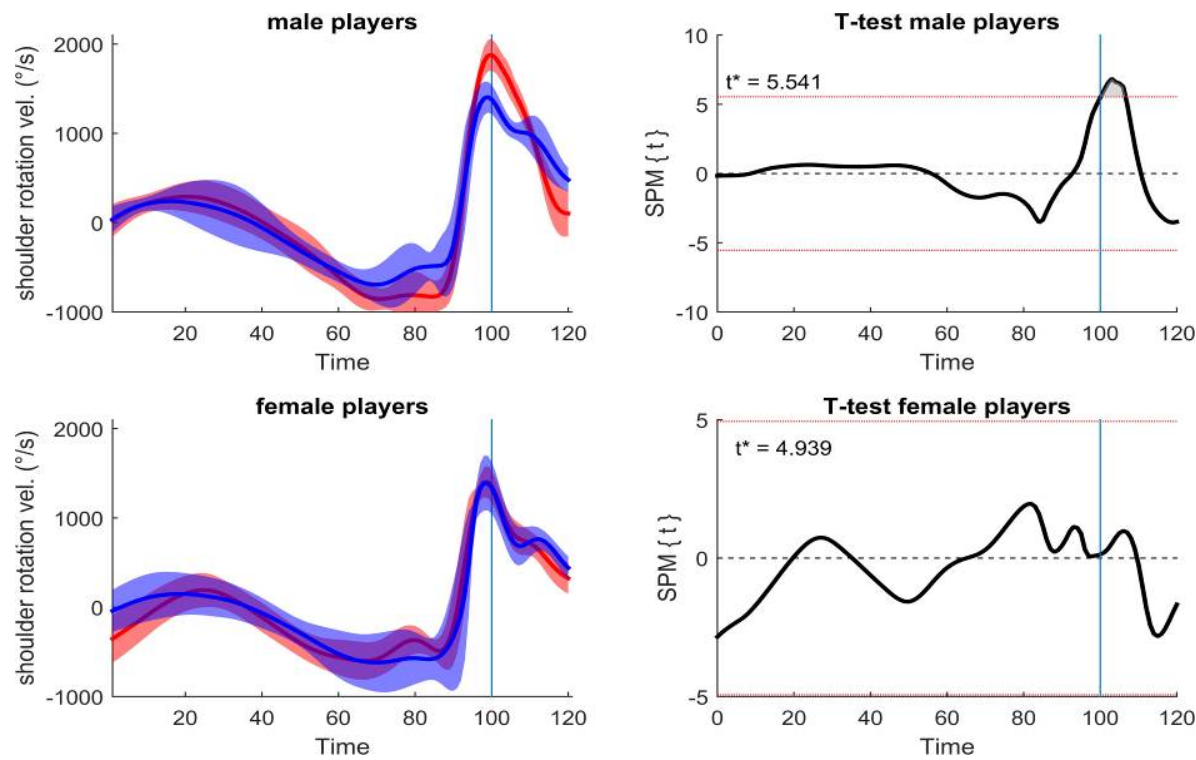

players however presented two supra-threshold clusters. The first one between $284 \mathrm{~ms}$ and $228 \mathrm{~ms}$ before ball impact ( $\mathrm{p}$ $10^{-5}$ ) and the second from ball impact until the end of the registered data $\left(\mathrm{p} \sim 10^{-7}\right)$. During both clusters, the top level players had a higher exorotation angle in the shoulder. Only at the moment of maximal exorotation (late cocking phase), no difference was observed in shoulder rotation.

There was a significant interaction effect for shoulder rotation velocity between $60 \mathrm{~ms}$ and $64 \mathrm{~ms}$ before ball impact ( $\mathrm{p}<0.00625$ ) and between $12 \mathrm{~ms}$ and $16 \mathrm{~ms}$ after ball impact $(p<0.00625)$. In Figure 5 are the post-hoc SPM t-tests for both genders. Just as for the shoulder rotation angle, no difference between levels of expertise was observed for the female players. In the case of the male players, significant higher shoulder endorotation velocities were seen for the top level players from ball impact until 28 $\mathrm{ms}$ later $\left(\mathrm{p} \sim 10^{-5}\right)$.
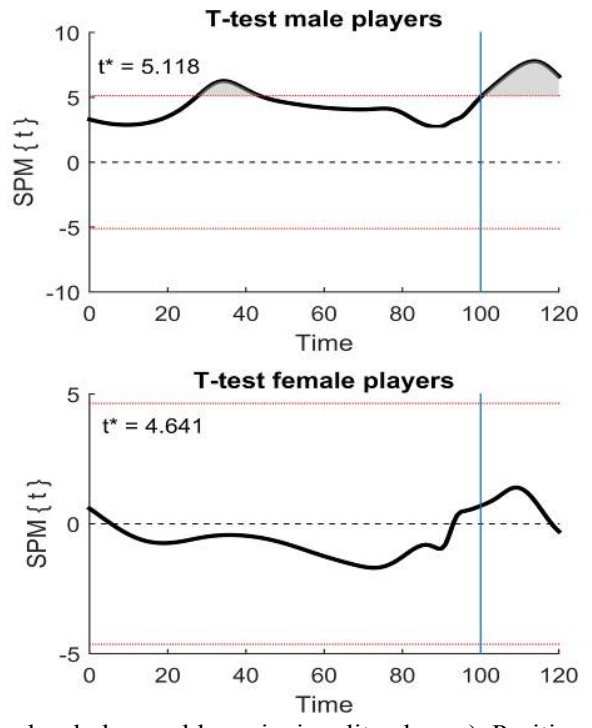

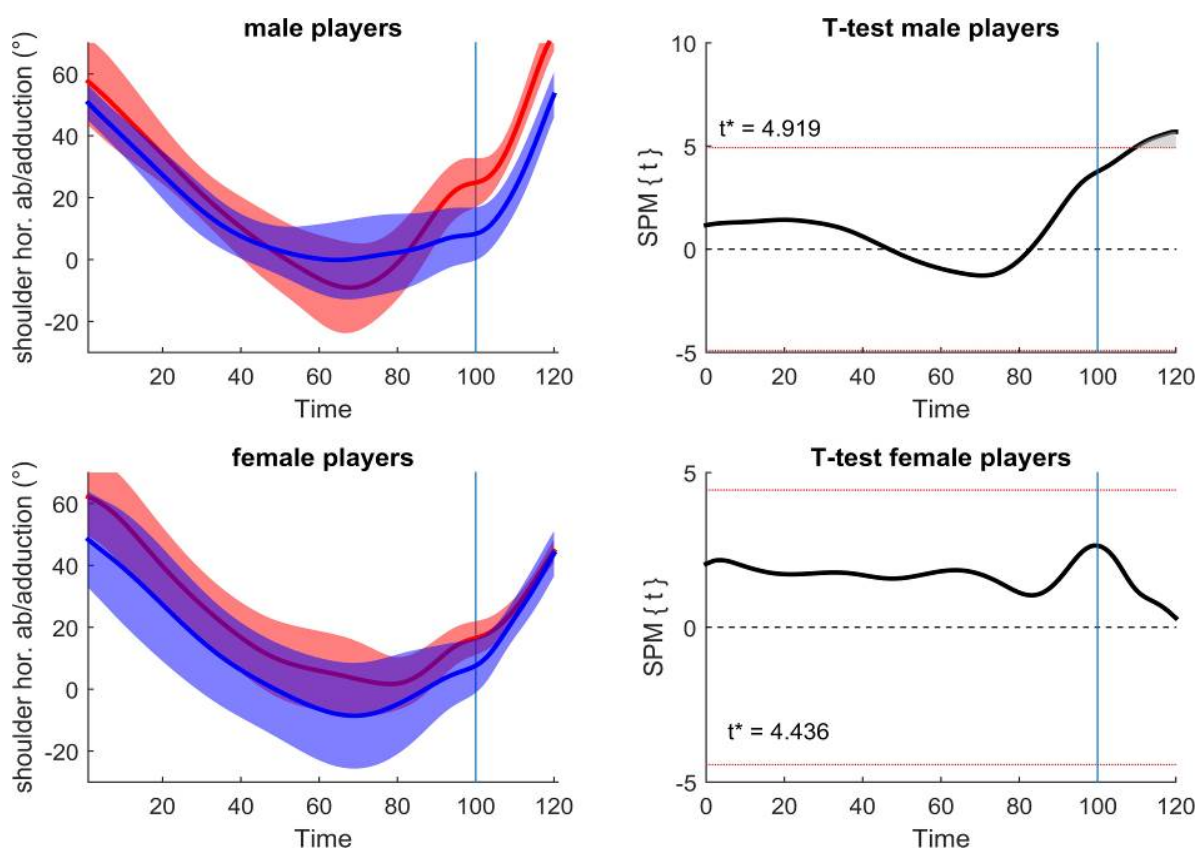

Figure 6. Post hoc T-tests on the shoulder horizontal ab/adduction angle time series (red $=$ top level players; blue $=$ junior elite players). Positive values are horizontal adduction (forward) and negative values are horizontal abduction (backward).
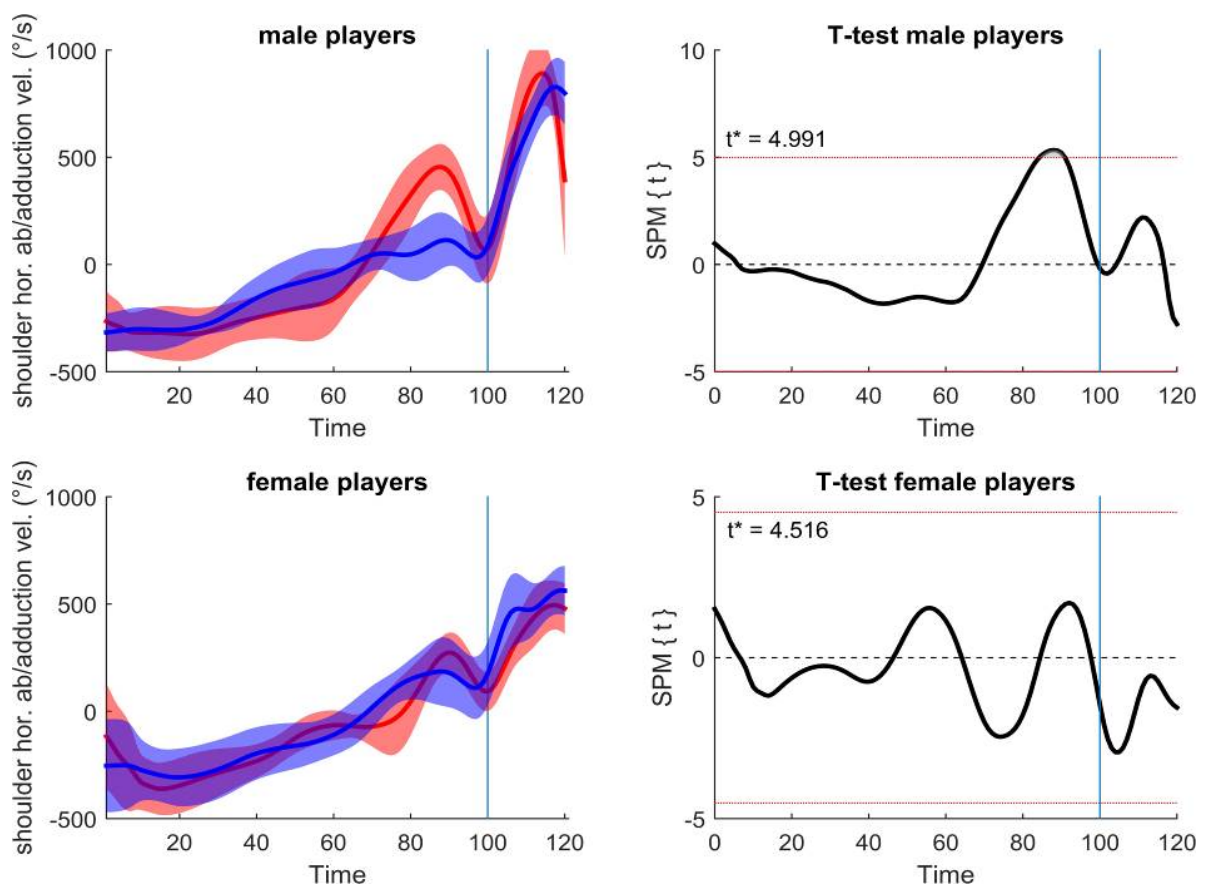

Figure 7. Post hoc T-tests on the shoulder horizontal ab/adduction velocity time series (red $=$ top level players; blue $=$ junior elite players). Positive values are adduction velocity and negative values are abduction velocity.

An interaction effect for shoulder horizontal ab/adduction occurred from $44 \mathrm{~ms}$ after ball impact until the end of the registered data $(\mathrm{p}<0.00625)$. Shown in Figure 6 are the post-hoc SPM t-tests for both genders. The time series for horizontal ab/adduction showed no difference between female top level and junior elite players. For the male players, a significant difference was observed from $20 \mathrm{~ms}$ after ball impact until the end of the registered data $(p=0.00092)$ during which the top level players' shoulders were more horizontally adducted.

Figure 7 shows the shoulder horizontal ab/adduction velocity. An interaction effect was seen from $88 \mathrm{~ms}$ until 56 $\mathrm{ms}$ before ball impact $(\mathrm{p}<0.00625)$. No effect of level of expertise was detected for the female players, but for the male players, a supra-threshold cluster was observed from 60 ms until $28 \mathrm{~ms}$ before ball impact $\left(\mathrm{p} \sim 10^{-4}\right)$. In this time-span, interesting behavior is seen for the male top level players: a short forward adduction velocity proceeded the point of impact, where after they froze this component of the shoulder motion and after ball impact, the forward adduction velocity followed a similar pattern like the others.

Figure 8 shows the SPM 2-way ANOVA on pelvis 
forward/backward tilt velocity. The interaction effect and main effect of expertise were not significant. A significant main effect of gender was located between 292 ms until 256
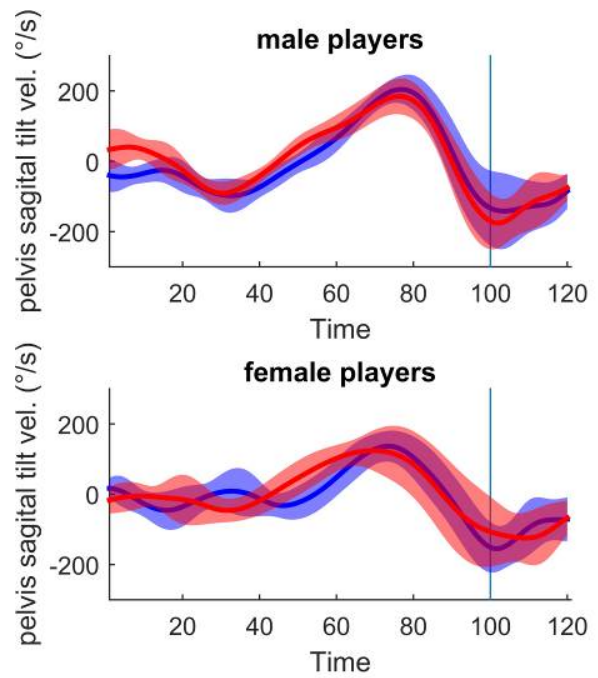

ms before ball impact, during which the male players had a little backward tilting motion while the female players' curves are around zero degrees per second.
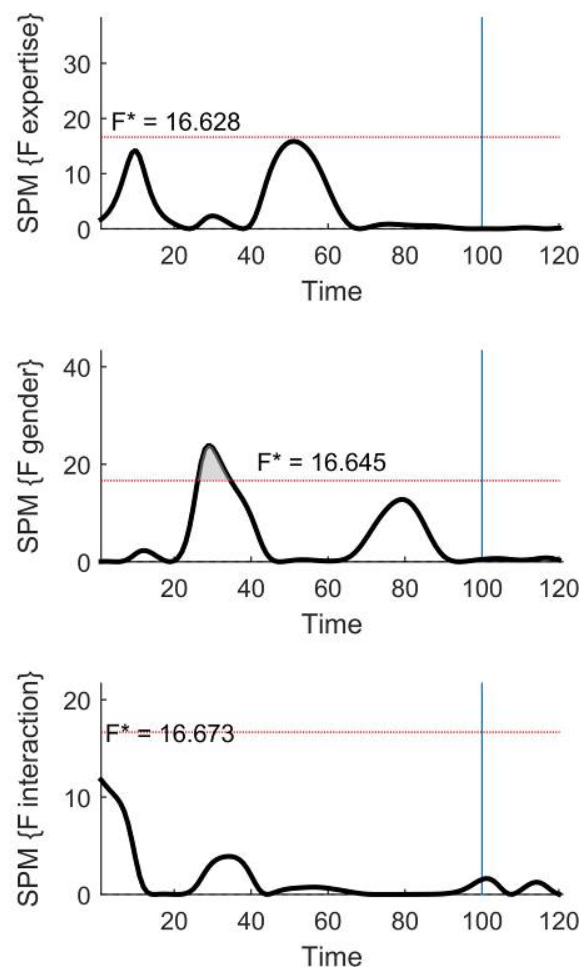

Figure 8. SPM two-way ANOVA on the pelvis forward/backward tilt angular velocity time series (red = top level players; blue = junior elite players). Positive values are forward tilt velocity and negative values are backward tilt velocity.
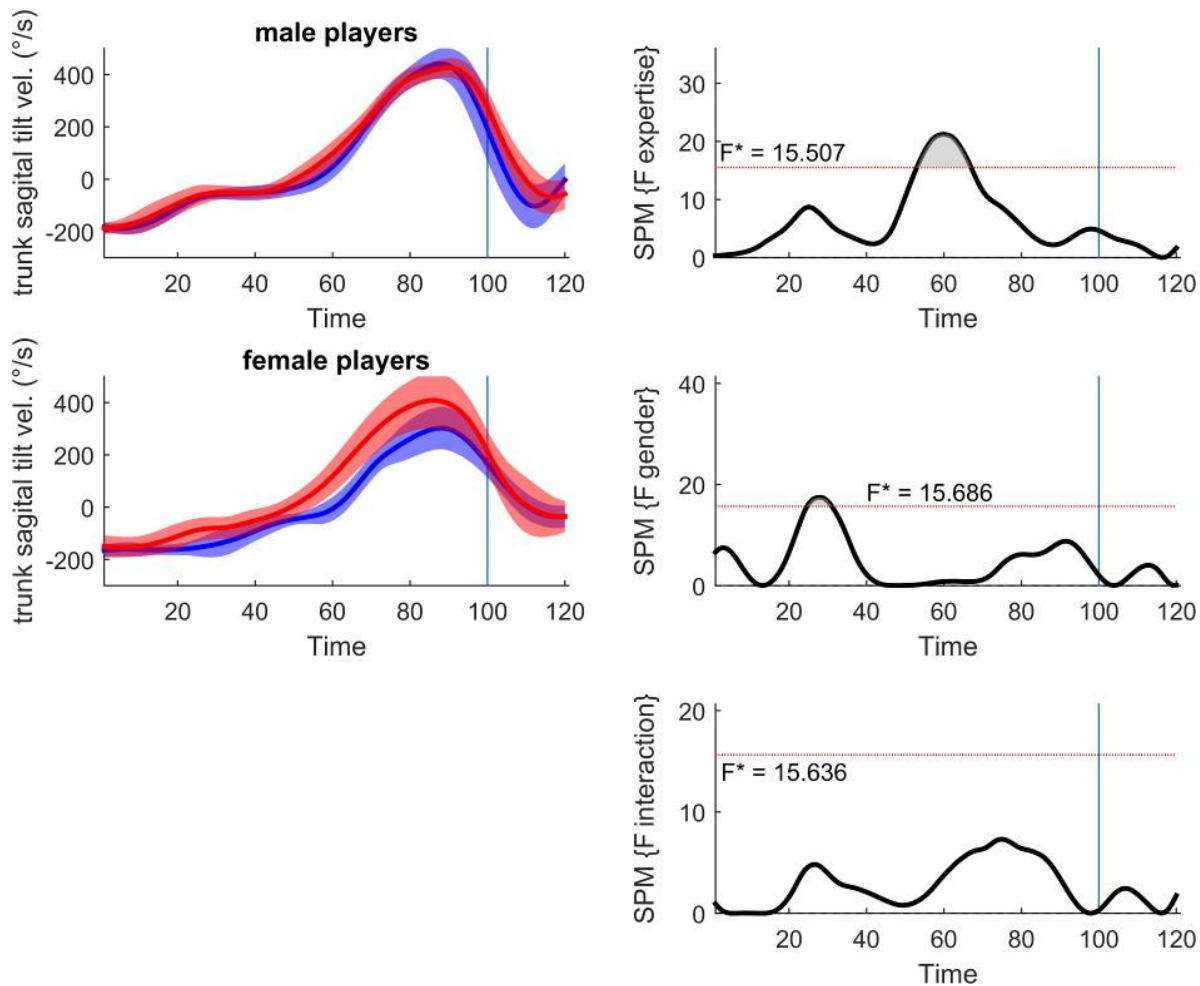

Figure 9. SPM two-way ANOVA on the trunk forward/backward tilt angular velocity time series (red = top level players; blue = junior elite players). Positive values are forward tilt velocity and negative values are backward tilt velocity. 
Just like for the forward/backward rotation velocity of the pelvis, the velocity of the trunk in the sagittal plane did not show any interaction effects (Figure 9). A significant supra-threshold cluster for expertise was seen from $184 \mathrm{~ms}$ until $128 \mathrm{~ms}$ before ball impact $(\mathrm{p}<0.00833)$. This time-span was positioned right before the maximal velocity was reached and during this period, the top level players showed a higher velocity. No significant difference was seen around the time of maximal velocity. The cluster for the main effect of gender was situated between $296 \mathrm{~ms}$ and 272 $\mathrm{ms}$ before ball impact $(\mathrm{p}<0.00833)$. During this time frame, the male players' curves was close to zero degrees per second, while the female curves were still negative, indicating that their trunks were still tilting backwards.

Figure 10 presents the SPM ANOVA results from the trunk rotation velocities. No interaction effect and no gender
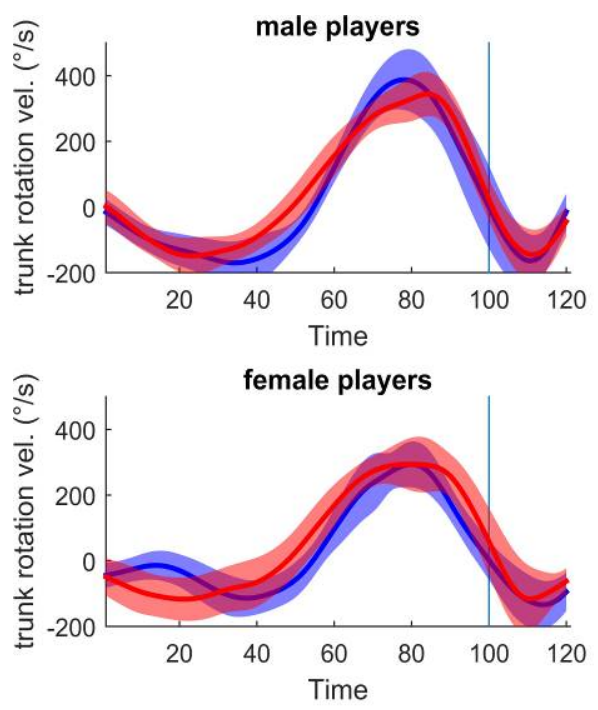

Figure 10. SPM two-way ANOVA on the trunk rotation velocity time series (red $=$ top level players; blue $=$ junior elite players). Positive values are rotation velocity toward the net.
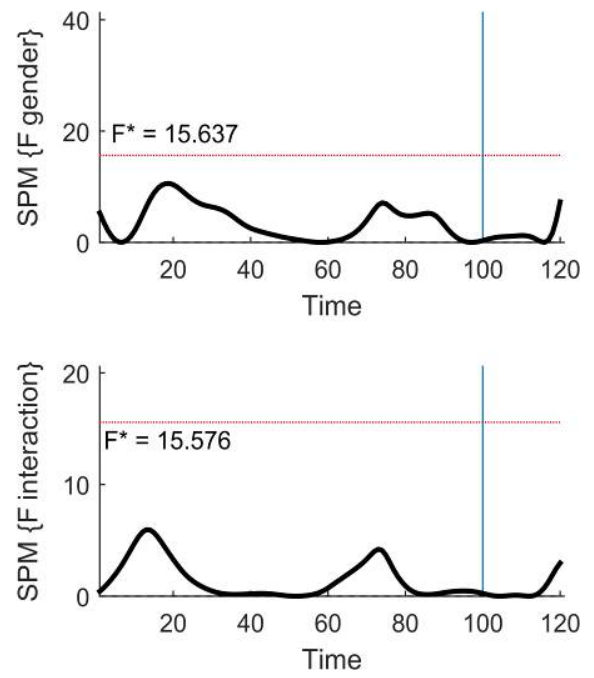

effect were present. The main effect of expertise was significant from $204 \mathrm{~ms}$ until $176 \mathrm{~ms}$ before ball impact $(\mathrm{p}<$ 0.00833 ), during which the top level players showed forward trunk rotation velocity while the junior elite players' curves were still negative or around zero. This indicated an earlier onset of trunk rotation in the top level players. No significant effects were observed around maximal trunk rotation velocity.

Figure 11 shows the results from the SPM ANOVA on the lateral tilting velocity of the trunk. At the start of the motion (400 ms until $396 \mathrm{~ms}$ before ball impact, $\mathrm{p}<0.00833$ ), an effect of expertise was present (top level players showed a higher velocity). A gender effect was observed around the time of ball impact ( $24 \mathrm{~ms}$ before until $16 \mathrm{~ms}$ after ball impact, $p<0.00833$ ). The female players' trunk lateral tilt velocity was significantly higher during this time span.

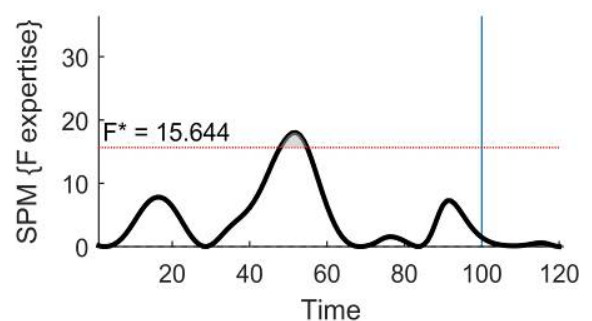



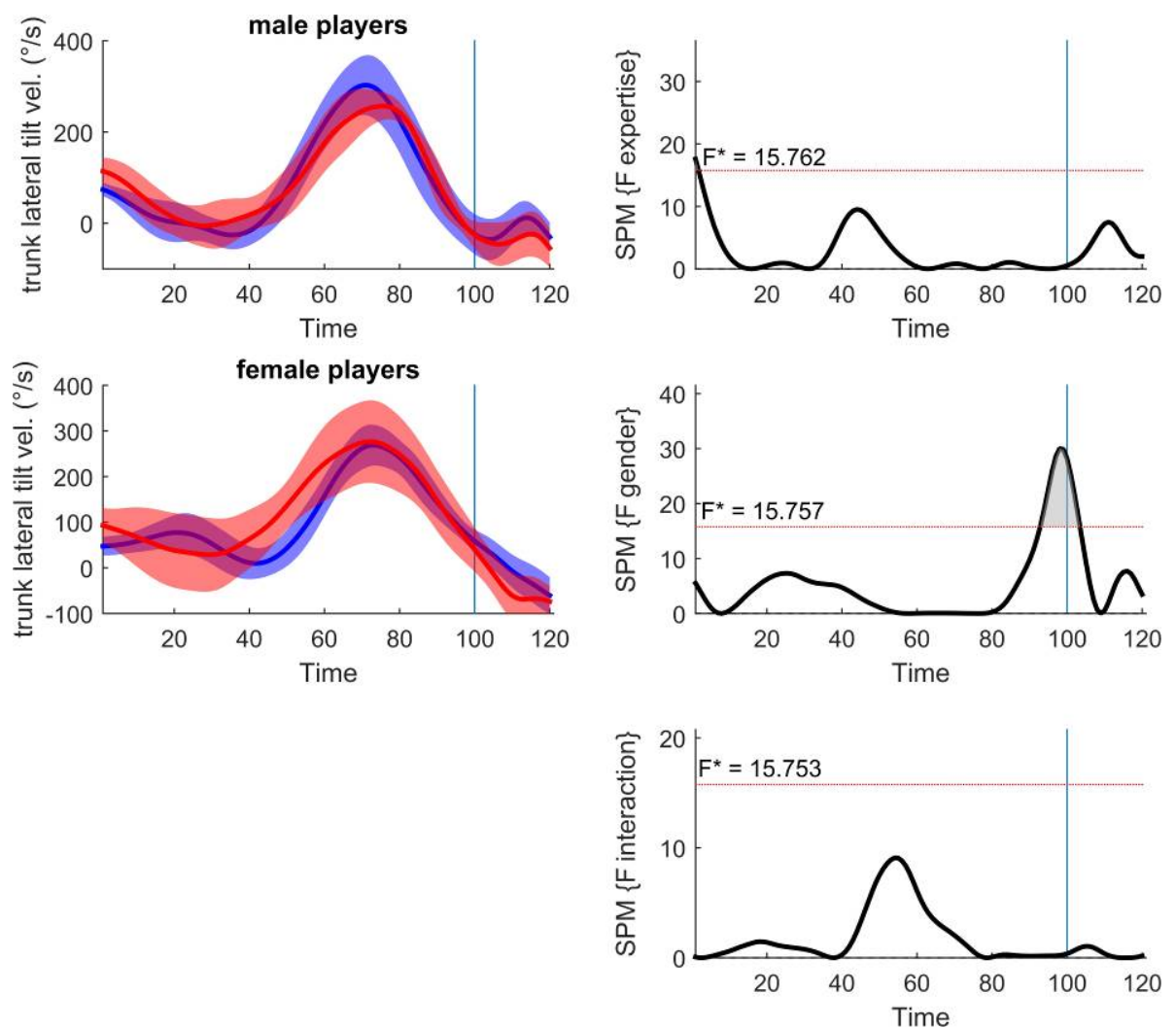

Figure 11. SPM two-way ANOVA on the trunk lateral tilt angular velocity time series (red $=$ top level players; blue $=$ junior elite players). Positive values are tilt velocity toward the contralateral side from the spike arm.

Finally, all ten variables that presented no significant main- or interaction effects are shown in Figure 12.
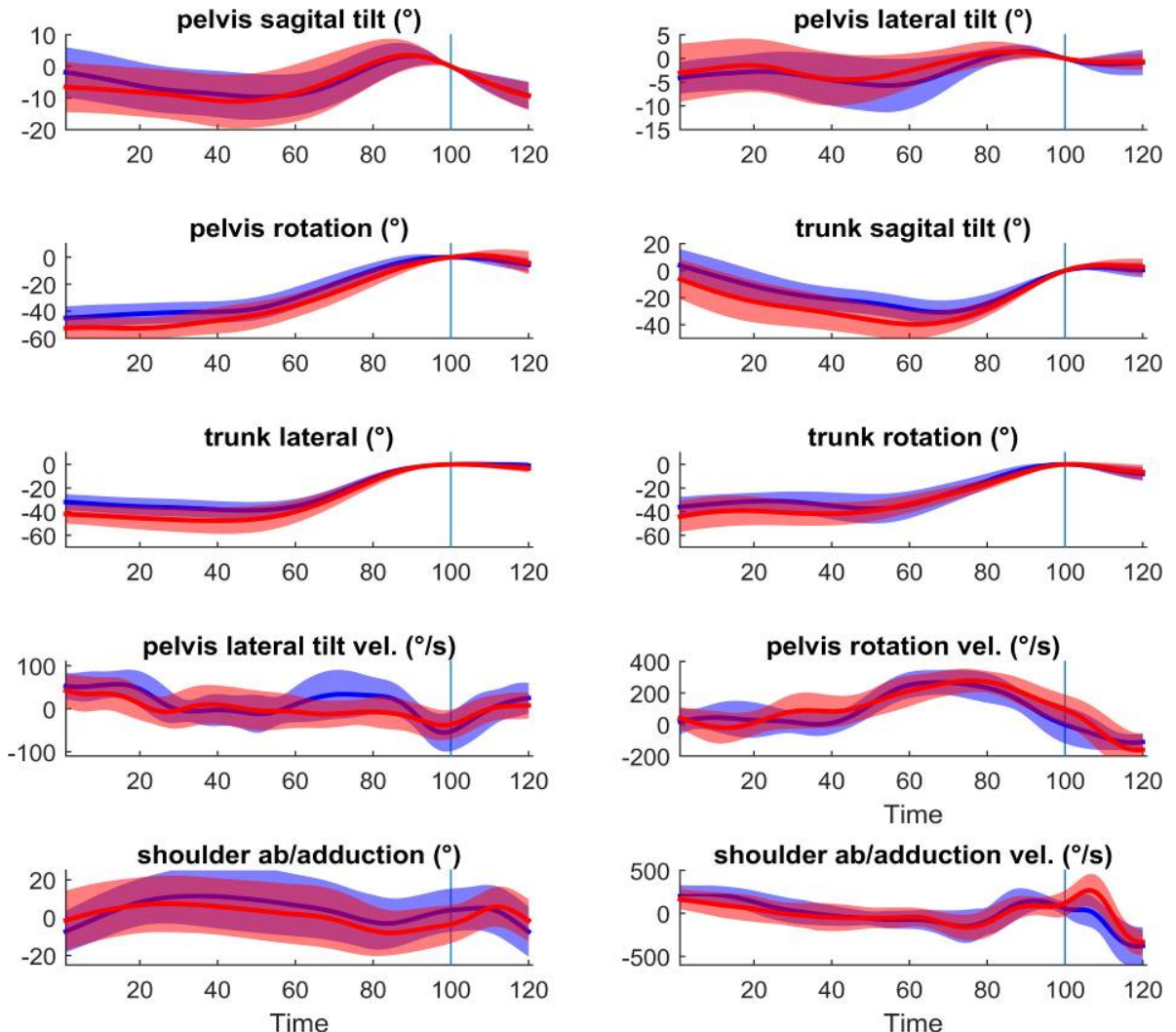

Figure 12. Kinematic time series that showed no significant main- or interaction effects (red $=$ top level players; blue $=$ junior elite players). The distinction between male and female players was not made to keep the figure clear. 


\section{Discussion}

In this paper, we analyzed differences in performance and three-dimensional kinematics during a volleyball spike aimed at a specific target. For this purpose, junior elite and professional male and female volleyball players were compared. As expected, impact speed of the hand was significantly higher for top level professional players than for the junior elite players. Also the significant main effect of gender is no surprise. The non-significant interaction effect between gender and level of expertise on impact speed suggests that both male and female junior elite players will increase their impact speed at a similar rate. The confirmation of this statement will have to wait until the longitudinal data of this study are analyzed. A limitation of this study is that we used hand impact speed and not the resulting ball speed. On the other side, hand impact speed is more under direct control of the motor control system than the ball speed. Also, the measurement of hand impact speed with the VICON system has a much higher accuracy than ball speed which has to be measured with a radar gun. The mean jump height from the top level male players in our study is comparable to the data from [11]. Jump height is an important parameter for optimal performance, because it enables an athlete to perform a good spike. Centeno-Prada et al. [18] advocated the use of jumping height as a good index of explosive strength in elite volleyball players. Also from a tactical viewpoint it is important to give a spiker time to adapt to a changing defense set-up from the other team and to be able to spike in a steep downward angle.

A first important observation about the kinematics is that the orientation angles for the pelvis and trunk in the global coordinate frame with respect to all three axes did not show any statistical significance (Figure 12). The MANOVA of these variables did not reach the threshold. This might suggest a set of invariant kinematic variables that remain constant during athletic development. Or it might be that the orientation angles of pelvis and trunk change in an earlier stadium. Our sample of junior volleyball athletes already attained a high stage of skill level. Perhaps the motor systems attempt to attain these specific orientation angles in the earliest stages of motor learning. The specific orientation angles observed during the spike motion indicate probably an optimal position for accelerating the more distal segments and remain therefore constant.

The MANOVAs on pelvis and trunk velocities and shoulder and elbow angles and velocities showed significant differences during several parts of the motion. Pelvis and trunk velocities presented mostly differences in the approach, cocking and acceleration phases and shoulder and elbow variables showed significant differences in the acceleration and follow-through phases. An interesting observation is that the pelvis and trunk velocities that were further analyzed with the two-way ANOVAs did not show significant interaction effects between gender and level of expertise. In contrast, the variables from the set of shoulder and elbow angles and angular velocities did show interaction effects (with elbow angle as an exception). These interaction effects might suggest that the male and female junior elite players' spike arm motions will go through a different development phase over the following years. Mostly non-significant differences were seen in the shoulder/elbow variables for the female players, only differences between the male players existed. The changing velocities of pelvis and trunk on the other hand appear to change in similar ways for both genders. Cools et al. [19] showed significant gender differences in strength of the shoulder muscles in a sample of (non-elite) volleyball players. We have not found similar evidence regarding core strength, but it is possible that differences in strength development contribute to the observed kinematic interaction effects.

Significant differences in pelvis and trunk velocities in the early parts of the motion are perhaps not related to impact speed differences directly but indirectly they have a large influence on the motion because of the sensitive dependence on initial conditions. Differences in the later parts of the motion reflect the importance of these variables to create high impact speeds. In the sagittal plane rotations of pelvis and trunk (Figures 8 and 9), two gender differences occurred around $280 \mathrm{~ms}$ before ball impact. The direction of the gender difference is reversed in pelvis and trunk sagittal rotations, indicating that the differentiated motions of these segments are different for both groups. Male players show a short backward pelvis tilt before starting to tilt forwards and female players keep the pelvis stable, while male players keep their trunk stable in the sagittal plane before tilting forwards and female players make the switch very fast from backward tilt to forward tilt. This difference could be a strategy learned from the lower air-time for female players or because of differences in the inertial properties of both segments. A main effect of expertise for trunk sagittal tilt velocity (Figure 9) occurred around $160 \mathrm{~ms}$ before ball impact during which the expert players were already tilting their trunk forwards, while the mean time series for the junior elite players were around zero degrees per second. This indicates an earlier onset of trunk forward tilt velocity for more experienced players. Also trunk rotation velocity (transverse plane) towards the net is initiated earlier in expert players (Figure 10), but no difference was observed around the maximal velocities. For the trunk lateral tilt (Figure 11), around the time of ball impact, the female players' trunks are still tilting to the contralateral side, while the male players' trunks are already tilting back to a straight posture.

There are some remarkable differences in the arm cocking and acceleration maneuvers in the spike motion. Top level players had an earlier onset of elbow extension and they hit the ball with a more extended elbow (Figure 2). A more extended elbow will put less strain on the shoulder when the shoulder internal rotation starts because the moment of inertia will be lower [20]. This same strategy is also seen in handball throwing and the tennis serve [10], [21]. The fact that the male top level players had the highest elbow extension velocity around impact (Figure 3) illustrates the importance of this part of the motion in generating a high 
impact speed. Another interesting observation in Figure 3 is the elbow flexion velocity of the male top level players after ball impact. Junior elite players and female top level players almost freeze elbow motion after impact, keeping the elbow extended. From a strategic point, quickly flexing the elbow back to normal (together with the rest of the body) allows the player to be in a prepared position more quickly to continue the game. The next difference in the arm cocking is seen in the shoulder rotation angles (Figure 4). Although the maximal shoulder exorotation was equal, the time series showed big differences between male top level and male junior players. This might suggest that the time-integrated cocking is more important for a fast recoil velocity (stretch-shortening-cycle of the rotator cuff muscles) than the maximal cocking. This could be an important conclusion, given that maximal exorotation is an important risk factor for shoulder injuries [22]. Indeed, the male top level players had a significantly greater shoulder endorotation velocity around the time of ball impact (Figure 5). This velocity was previously shown to be correlated to ball speed in volleyball [3], but also in team-handball throwing [23]. Shoulder horizontal ab/adduction (Figure 6) is also an important component of the cocking and acceleration of the spike arm. Although it was not statistically significant, male top level players and female junior players had a small shoulder horizontal abduction, thereby stretching the pectoralis muscle whereas the other groups kept their arms in a constant horizontal adducted position throughout the motion. The horizontal adduction was significantly higher for the top level male players in the follow through phase. Their total range of horizontal shoulder adduction is thus larger and gives them a bigger path whereupon to accelerate the spike hand. The pattern of horizontal adduction - freeze horizontal adduction observed for the male top level players (Figure7) creates a large horizontal abduction acceleration right before ball impact. This will cause the forearm to start rotating by an inertial effect. Apparently they have developed an efficient inertial propelling mechanism of the forearm instead of actively extending the elbow.

The observed main and interaction effects suggest that male and female players use different mechanisms for generating a high impact speed and that the junior elite players go through age-gender specific developmental pathways. This information could be important in constructing age-gender specific training programs for coordination and strength. Knowing the mechanisms used by different groups allows focusing the training better on the required muscle actions. Knowledge of age and gender specific motion patterns in the volleyball spike were still lacking from the literature.

Statistical Parametric Mapping has a big advantage over classical $0 \mathrm{D}$ statistical testing. Using this relatively new technique, we were able to highlight several important differences in the mechanics of the spike motion between genders and levels of expertise. Pataky et al. [13] have shown that extracting extrema can introduce a bias in the analysis. Indeed, in our data-set, many differences were not located around local or global extrema. Thus without introducing a multiple testing problem, SPM allows to compare entire time series. However, a multiple testing problem still persists in the number of variables that we used $(n=20)$ that is not controlled by SPM. The post-hoc procedures that the spm-1d package currently has available are likely too simple (www.spm1d.org, (C) T.C. Pataky). Bonferroni corrections to the post-hoc tests alpha level were performed, but this assumes that all variables are independent from each other which are clearly not the case in a multi-link kinematic chain. Thus, our analysis was rather conservative and more differences (more and longer threshold up crossings in more variables) are very likely with more appropriate procedures. Post-hoc procedures following MANOVA like descriptive discriminant analysis [24] are more appropriate but currently not available for SPM. Within this limitation, the current approach with SPM is still useful in generating hypotheses about the longitudinal development. Future studies could also perform EMG measurements concurrently with kinematics and incorporate body seize parameters as covariates into the statistical model. This could give an even higher insight into the biomechanical process of performing a complex motion like the volleyball spike.

A limitation that still persists in this kind of analysis is the group-averaging process that ignores typical mechanical patterns that are very individual-specific [25]. Case studies about the links between biomechanics and performance parameters could overcome this. The variance within and between players is interpreted as noise in the general linear model. Research has shown that this variance is important and deserves a study of its own [26], [27]. Therefore, part two of this study will focus more on that aspect and will use individually-based data analyses.

\section{Conclusions}

Performance and 3D-kinematics of the volleyball spike were analyzed for junior elite and professional volleyball players from both genders. In both performance parameters (hand impact speed on the ball and jumping height), significant main effects of level of expertise were demonstrated in favor of the professional athletes and significant main effects of gender in favor of the male players. The 3D-kinematics were contrasted between the groups with a relatively new technique, Statistical Parametric Mapping, that allowed us to detect specific differences throughout the entire motion that would have been missed by classical techniques. Specific differences were observed in the angular velocities of pelvis and trunk and orientation and velocity of shoulder and elbow. These differences illustrate the different mechanisms that are used in generating a high impact speed. Interestingly, the pelvis and trunk velocities showed almost no interaction effects between gender and level of expertise, while the variables from shoulder and elbow did. This might suggest different learning routes followed by both genders. A confirmation of 
that suggestion will have to wait for the results of the longitudinal follow-up.

\section{Acknowledgements}

The authors wish to thank all players for their participation in this study, Brecht Van Kerckhove for coordinating with the Topsportschool and Louis Curtil, Eline De Cleyn and Charlotte Genbrugge who were involved in the project for their Master thesis.

\section{REFERENCES}

[1] A. Kugler, M. Kruger-Franke, S. Reininger, H. H. Trouillier, and B. Rosemeyer, "Muscular imbalance and shoulder pain in volleyball attackers," Br. J. Sports Med., vol. 30, pp. 256-259, 1996.

[2] J. C. Reeser, G. S. Fleisig, B. Bolt, and M. Ruan, "Upper limb biomechanics during the volleyball serve and spike.," Sports Health, vol. 2, no. 5, pp. 368-74, Sep. 2010.

[3] S. G. Coleman, A. S. Benham, and S. R. Northcott, "A three-dimensional cinematographical analysis of the volleyball spike.," J. Sports Sci., vol. 11, no. 4, pp. 295-302, 1993.

[4] J. R. Brown, J. Alsarraf, Bader, M. Waller, P. Eisenman, and C. A. Hicks-Little, "Rotational Angles and Velocities During Down the Line and Diagonal Across Court Volleyball Spikes," Int. J. Kinesiol. Sport. Sci., vol. 2, no. 2, pp. 1-8, 2014.

[5] B. Forthomme, J. L. Croisier, G. Ciccarone, J. M. Crielaard, and M. Cloes, "Factors Correlated With Volleyball Spike Velocity," Am. J. Sports Med., vol. 33, no. 10, pp. 1513-1519, 2005.

[6] A. S. Rokito, F. W. Jobe, M. M. Pink, J. Perry, and J. Brault, "Electromyographic analysis of shoulder function during the volleyball serve and spike," J. Shoulder Elb. Surg., vol. 7, no. 3, pp. 256-263, 1998.

[7] L. Mitchinson, A. Campbell, D. Oldmeadow, W. Gibson, and D. Hopper, "Comparison of upper arm kinematics during a volleyball spike between players with and without a history of shoulder injury.," J. Appl. Biomech., vol. 29, no. 2, pp. 155-64, Apr. 2013.

[8] M. Tilp, H. Wagner, and E. Müller, "Differences in 3D kinematics between volleyball and beach volleyball spike movements.," Sport. Biomech., vol. 7, no. 3, pp. 386-97, Sep. 2008.

[9] E. Seminati, A. Marzari, O. Vacondio, and A. E. Minetti, "Shoulder 3D range of motion and humerus rotation in two volleyball spike techniques: injury prevention and performance," Sport. Biomech., vol. 14, no. July, pp. 1-16, 2015.

[10] H. Wagner, J. Pfusterschmied, M. Tilp, J. Landlinger, S. P. von Duvillard, and E. Müller, "Upper-body kinematics in team-handball throw, tennis serve, and volleyball spike.," Scand. J. Med. Sci. Sports, pp. 1-10, Jul. 2012.

[11] H. Wagner, M. Tilp, S. P. von Duvillard, and E. Mueller,
"Kinematic analysis of volleyball spike jump.," Int. J. Sports Med., vol. 30, no. 10, pp. 760-5, Oct. 2009.

[12] S. H. Strogatz, Nonlinear Dynamics and Chaos - with applications to Physics, Biology, Chemistry and Engineering., 2nd ed. Boulder, Colorado: Westview Press, 2015.

[13] T. C. Pataky, M. A. Robinson, and J. Vanrenterghem, "Vector field statistical analysis of kinematic and force trajectories," $J$. Biomech., vol. 46, no. 14, pp. 2394-2401, 2013.

[14] L. Z. F. Chiu and G. J. Salem, "Pelvic kinematic method for determining vertical jump height," J. Appl. Biomech., vol. 26, no. 4, pp. 508-511, 2010.

[15] V. Zatsiorsky, Kinematics of Human Motion. Human Kinetics, Leeds UK, 1998.

[16] K. Friston, J. Ashburner, S. Kiebel, T. Nichols, and W. Penny, Statistical Parametric Mapping - The Analysis of Functional Brain Images. Amsterdam: Elsevier, 2007.

[17] R. J. Adler and J. E. Taylor, Random Fields and Geometry. New York: Springer-Verslag, 2007.

[18] R. A. Centeno-Prada, C. Lopez, and J. Naranjo-Orellana, "Jump percentile: A proposal for evaluation of high level sportsmen," J. Sports Med. Phys. Fitness, vol. 55, no. 5, pp. 464-470, 2015.

[19] A. M. J. Cools, F. Vanderstukken, F. Vereecken, M. Duprez, K. Heyman, N. Goethals, and F. Johansson, "Eccentric and isometric shoulder rotator cuff strength testing using a hand-held dynamometer: reference values for overhead athletes," Knee Surgery, Sport. Traumatol. Arthrosc., 2015.

[20] D. a Hong, T. K. Cheung, and E. M. Roberts, "A three-dimensional, six-segment chain analysis of forceful overarm throwing.," J. Electromyogr. Kinesiol., vol. 11, no. 2, pp. 95-112, Apr. 2001.

[21] B. Serrien, R. Clijsen, J. Blondeel, M. Goossens, and J.-P. Baeyens, "Differences in ball speed and three-dimensional kinematics between male and female handball players during a standing throw with run-up," BMC Sports Sci. Med. Rehabil., vol. 7, no. 27, 2015.

[22] K. E. Wilk, K. Meister, and J. R. Andrews, "Current Concepts in the Rehabilitation of the Overhead Throwing Athlete.," Am. J. Sport. Med., vol. 30, no. 1, pp. 136-151, 2002.

[23] H. Wagner, J. Pfusterschmied, S. P. von Duvillard, and E. Müller, "Performance and kinematics of various throwing techniques in team-handball," J. Sport. Sci. Med., vol. 10, pp. 73-80, 2011.

[24] M. Barton, P. E. Yeatts, R. K. Henson, and S. B. Martin, "Moving Beyond Univariate Post-Hoc Testing in Exercise Science: A Primer on Descriptive Discriminate Analysis," Res. Q. Exerc. Sport, pp. 1-11, Aug. 2016.

[25] P. Glazier, "Game, Set and Match? Substantive Issues and Future Directions in Performance Analysis.," Sport. Med., vol. 40, no. 8, pp. 625-634, 2010.

[26] S. J. A. Kelso, Dynamic Patterns. The self-organization of brain and behaviour. Cambridge, MA: MIT Press, 1995.

[27] E. Preatoni, J. Hamill, A. J. Harrison, K. Hayes, R. E. a. Van Emmerik, C. Wilson, and R. Rodano, "Movement variability and skills monitoring in sports," Sport. Biomech., no. April 2013, pp. 1-24, Nov. 2012. 